\title{
Ein Versuch die Rotationsgeschwindigkeit des Venusaequators auf spectrographischem Wege zu bestimmen.
}

\author{
Von A. Belopolsky.
}

Seit dem 25. März d. J. benutzte ich die günstige Stellung der Venus zu photographischen Aufnahmen ibres Spectrums. Bis zum Mai wurde dazu ein Zweiprismen-Spectrograph, dann einer mit drei Compoundprismen benutzt; beide Spectrographen wurden am 30 zöll. Refractor angebracht.

Die ersten Aufnahmen schienen mir so vorzüglich zu sein, dass ich es unternahm zu untersuchen, ob auf ihnen der Einfluss einer kurzen Rotationsperiode zu betnerken wäre. Die Hoffnung dies auf den Spectrogrammen messen zu können wurde noch dadurch vermehrt, dass Venus mit reflectirtem Lichte leuchtet, dass also die Verschiebung der Spectrallinien in Folge der Rotation der doppelten Rotationsgeschwindigkeit entspricht. Bei voller Beleuchtung des Planeten ist demnach die der relativen Verschiebung der Spectrallinien an den Rändern des Venusspectrums entsprechende Geschwindigkeit gleich der vierfachen wahren Geschwindigkeit; und wenn also die Geschwindigkeit des Venusaequators $=0.5 \mathrm{~km}$ pro Secunde ist (bei kurzer Rotationsperiode), so zeigen die Verschiebungen der Spectrallinien eine Geschwindigkeit von $2 \mathrm{~km}$ pro Secunde an.

Zur Zeit als ich meine Beobachtungen begann, konnte man noch eine gegenseitige Verschiebung der Spectral linien an den Rändern des Spectrums erwarten, die einer relativen Geschwindigkeit von $\mathrm{I} 1 / 2 \mathrm{~km}$ pro Secunde entspricht. Wenn diese Grösse mittelst des hiesigen Spectrographen auch nicht genau gemessen werden kann, so kann doch der Sinn der Verschiebung wohl bemerkt werden. Nehmen wir nach Schiaparelli und Trouvelot an, dass die Rotationsaxe der Venus einen Winkel von $78^{\circ}-80^{\circ}$ mit der Bahnebene bildet, so kann der Einfachheit wegen folgende Relation zwischen spectroskopischer und Rotationsgeschwindigkeit angenommen werden:

$$
w={ }_{4} \cos ^{2} 1 / 2 \alpha \cdot v=k v,
$$

wo $a$ den Winkel Erde-Venus-Sonne bedeutet.

Für $\alpha$ und $k$ und den Venusradius ergeben sich die folgenden Werthe:

$\begin{array}{rccc}\text { 1900 } & \alpha & k & \text { Venusradius } \\ \text { März 25 } & 70^{\circ} 29^{\prime} & 2.7 & 8.6 \\ \text { April 8 } & 7721 & 2.4 & 9.5 \\ 22 & 8824 & 2.1 & 11.0\end{array}$

Um die Genauigkeit möglichst zu erhöhen, benutzte ich Platten mit äusserst feinem Silberniederschlag, sogar so wenig empfindliche, wie die Thomas'schen Lantern-Platten. Diese haben aber den Nachtheil, dass die Expositionszeit sehr verlängert werden muss, und dass das künstliche Spectrum erst nach der Exposition auf den Planeten aufgenommen wurde.

Auf einigen Platten wurden zwei Aufnahmen gemacht.

Zur Einstellung des Planeten auf den Spalt des Spectrographen diente das am 30 Zöller angebrachte Leitrohr von
40 Fuss Brennweite und 400 facher Vergrösserung. Der Spalt schnitt den Randbogen des Planeten in der Mitte. Zur Controlle des Instruments wurden auch Aufnahmen nach einer Drehung des Spalts um 90 Grad gemacht, indem der Spalt auf den Terminator eingestellt wurde.

Die Messungen wurden mittelst des Mikroskops von Töpfer unter Anwendung einer $15 \mathrm{f}$. bis $40 \mathrm{f}$. Vergrösserung ausgeführt. Ich muss hierbei bemerken, dass eine zu starke Vergrösserung bei mir einen bestimmten persönlichen Fehler verursacht.

Die Messungen wurden auf zweierlei Arten durchgeführt. Entweder wurde der Faden auf die Ränder des Spectrums eingestellt und die Trommel abgelesen, oder es wurden die Winkel gemessen, welche die künstlichen Linien mit denen des Venusspectrums bilden. Zu diesem $Z_{\text {weck }}$ ist vom hiesigen Mechaniker am Ocular des Mikroskops ein kleiner Positionskreis angebracht. Grösstentheils benutzte ich die letztere Methode.

Bezeichnet man den Winkel zwischen den künstlichen und den Venuslinien mit $\varphi-\varphi_{0}$, und mit $R$ den linearen Radius der Venus in der Ebene der Cassette, so ist die Verschiebung $d$

$$
d=2 \Delta=R \sin \left(\varphi-\varphi_{0}\right),
$$

wo $\Delta$ die der Aequatorgeschwindigkeit entsprechende Verschiebung bedeutet.

Wenn der w. F. des Mittels von $\varphi-\varphi_{0}$ aus 10-15 Spectrallinien \pm 0.05 beträgt, so wäre für $2 \Delta$ der w. F. $= \pm 0.5 \mathrm{~km} z u$ erwarten, und man könnte dann schon den Einfluss der Rotation bemerken, da, wie schon gesagt, unter der Annahme einer Geschwindigkeit des Venusaequators von $0.5 \mathrm{~km}$ pro Secunde, die der gegenseitigen Verschiebung der Spectralränder entsprechende relative Geschwindigkeit zur Zeit meiner ersten Beobachtung noch $1.5 \mathrm{~km}$ betragen konnte. Im Durchschnitt wird der w. F. von $\varphi-\varphi_{0}= \pm 0.07$ resp. $\pm 0.6 \mathrm{~km}$ pro Secunde. Dabei muss man annehmen, dass das Instrument keine constanten Fehler von derselben Grösse besitzt. Um den Untersuchungen mehr Gewicht zu geben, benutzte ich seit Anfang Mai einen Spectrographen mit drei Rutherfurd'schen Prismen und ich habe damit fünf messbare Spectrogramme erhalten. Der w. F. von $2 \Delta$ wird bei diesem Instrument $= \pm 0.3 \mathrm{~km}$ pro Secunde, also ist es für die Entscheidung der uns interessirenden Frage geeigneter; jedoch erschwette die starke Krümmung der Spectrallinien die Messungen.

Beim Ausmessen legte ich die Platten immer so, dass das rothe Ende des Spectrums links lag. Dann ist der obere Rand im Mikroskop der Westrand des Planeten. Die Ablesung des Positionskreises nimmt ab im Sinne von Violett gegen Roth; wenn also die Venuslinien mit den künstlichen einen positiven Winkel bilden, so ist bei Einstellung auf die Venuslinien die Ablesung kleiner als bei der auf die künstlichen Linien. 
März 25*) Zwei Aufnahmen auf einer Platte von je 20 Minuten. Gute Platte.

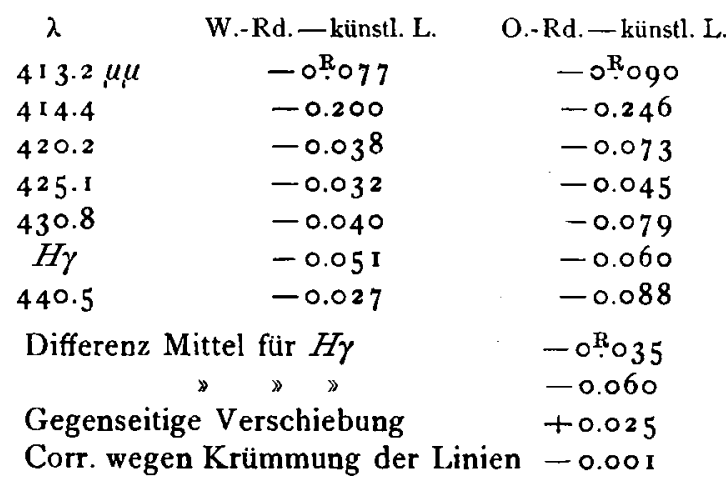

$2.7 v=4.3 \mathrm{~km} \quad v=+1.6 \mathrm{~km}$ pro Secunde.

Dieselbe Platte wurde nochmals ausgemessen; hierbei wurden die Differenzen der Einstellungen auf die Ränder folgendermaassen bestimmt :

Gruppe $\left\{\begin{array}{cc}\lambda & \text { Diff. W. }- \text { O. Rd. Venus-L. } \\ 4 \text { I } 3.2 \mu \mu & +0.015 \\ 4 \text { I } 4.4 & +0.021 \\ 417 & +0.008 \\ 418 & +0.016 \\ 420.2 & +0.003 \\ 424 & +0.012 \\ 425.1 & +0.026 \\ 429.5 & +0.012 \\ 430.8 & +0.001 \\ H \gamma & +0.036 \\ 435.2 & -0.013 \\ 443.5 & +0.005 \\ \text { Mittel } & +0.002\end{array}\right.$

$\lambda$ Diff. W. - O. Rd. künstl. L.

$\begin{array}{cc}413.2 \mu \mu & +0.002 \\ 414.4 & -0.015 \\ 420.2 & -0.023 \\ 425.1 & -0.001 \\ 427.2 & -0.020 \\ 430.8 & -0.003 \\ 432.6 & -0.010 \\ H \gamma & -0.022 \\ 440.5 & -0.009 \\ \text { Mittel } & -0.011\end{array}$

Verschiebung $(\mathbf{W}-\mathbf{O})$ Rand $=+0^{\mathbf{R}} .2_{2}$

$2.7 v=3.5 \mathrm{~km} \quad v=+1.3 \mathrm{~km}$ pro Sec.

März 3o. I. Platte, sehr gut, Expos.-Zeit 60 Minuten.

$\begin{array}{cc}\lambda & \text { Pos. }-K r . \text { Venus-L. } \\ 424.7 \mu \mu & \text { I } 3.5 \\ 425.1 & 12.2 \\ 425.4 & 13.3 \\ 429.5 & \text { I } 2.5 \\ 431.4 & \text { I } 2.7\end{array}$

*) Etwas zu viel entwickelt.

$\begin{array}{cc}\lambda & \text { Pos. }- \text { Kr. Venus }-\mathrm{L} . \\ 431.5 \mu \mu & 12: 9 \\ 435.2 & 13.2 \\ 452.6 & 13.1 \\ \lambda & \text { Pos. - Kr. künstl. L. } \\ 425.1 \mu \mu & \text { I } 3.05 \\ 427.2 & 13.03 \\ 430.8 & 12.92 \\ 432.6 & 12.95 \\ 438.4 & 12.92 \\ 440.5 & 12.90\end{array}$

Mittel für Venus-Linien $=12 \% 91$ Mittei für künstliche Linien $=12.96$ $\varphi-\varphi_{0} \quad=+0.05$ $2 v=+0.5 \mathrm{~km} \quad v=+0.2 \mathrm{~km}$.

Zweite Messung derselben Platte.

\begin{tabular}{|c|c|}
\hline$\lambda$ & Pos.-Kr. Venus - L \\
\hline $407.7 \mu \mu$ & $12: 32$ \\
\hline 407.8 & $12.5^{8}$ \\
\hline $4 I 1.0$ & 12.40 \\
\hline 411.9 & I 1.74 \\
\hline 414.4 & I 2. I 4 \\
\hline $4 \cdot 5.2$ & 12.08 \\
\hline 418.2 & 12.04 \\
\hline 418.8 & 11.74 \\
\hline 419.9 & I 2.18 \\
\hline 421.6 & 12.00 \\
\hline 423.4 & 11.90 \\
\hline 423.9 & J 2.20 \\
\hline$\lambda$ & Pos.-Kr. künstl. L. \\
\hline 4 I $3.2 \mu \mu$ & $12: 52$ \\
\hline 414 & $12.5^{8}$ \\
\hline $4^{20} 0.3$ & 12.52 \\
\hline 425.1 & 1262 \\
\hline 427.2 & I 2.48 \\
\hline 430.8 & 12.50 \\
\hline
\end{tabular}

Mittel für Venus-Linien $=12 \% 4$ Mittel für künstliche Linien $=12.54$ $\varphi-\varphi_{0} \quad=+0.5^{\circ}$

für die Gegend $\lambda=420 \mu \mu$

$$
2 v=+2.6 \mathrm{~km} \quad v=+1.3 \mathrm{~km} \text {. }
$$

März 30. 2. Platte, Exp.-Z. 7 Min., sehr gute Platte.

$\begin{array}{cc}\lambda & \text { Diff. W. }- \text { O.Rd.Venus-L. } \\ 423.6 \mu \mu & -0.021 \\ 424.0 & -0.013 \\ 424.3 & -0.003 \\ 428.6 & +0.011 \\ 429.5 & -0.010 \\ 429.7 & 0.000 \\ 430.7 & -0.020 \\ 431.4 & -0.004 \\ 431.5 & -0.013 \\ H \gamma & -0.002 \\ 435.2 & -0.012 \\ 436.0 & -0.016 \\ 437.1 & -0.021\end{array}$


$\lambda \quad$ Diff. W. - O. Rd. Venus-L.

$\begin{array}{ll}439.5 \mu \mu & -0.022 \\ 443.5 & -0.010 \\ 446.5 & -0.001 \\ \text { Mittel } & -0.010\end{array}$

$\lambda$

$427.2 \mu \mu$

430.8

432.6

438.4

440.5

Mittel

Diff. W. - O. Rd. künstl. L.

$$
\begin{aligned}
& -0.022 \\
& -0.012 \\
& -0.007 \\
& -0.015 \\
& -0.019 \\
& -0.015
\end{aligned}
$$

Verschiebung ( $\mathbf{W}-\mathbf{O})$ Rand $=+0 \stackrel{\mathbf{R}}{005}$ Corr. für Krümm. der Linien $=+0.00 \mathrm{I}$ $2.7 v=1.1 \mathrm{~km} \quad v=+0.4 \mathrm{~km}$ pro Sec.

April 4. Expositionszeit 6o Minuten. Sehr schwache künstliche Linien.

$$
\lambda
$$

$424.5 \mu \mu$
424.8
429.5
430.7
431.4
431.5
436.0
437.1
439.5
442.7
445.9
446.9
447.6

$\lambda$

$$
\begin{aligned}
& 427.2 \mu \mu \\
& 430.8 \\
& 432.6 \\
& 438.4
\end{aligned}
$$$$
\text { Diff. W. - O. Rd. Venus-L. }
$$

$$
\begin{aligned}
& -0.020 \\
& -0.028 \\
& -0.008 \\
& -0.019 \\
& -0.042 \\
& -0.026 \\
& -0.023 \\
& -0.010 \\
& -0.012 \\
& -0.008 \\
& -0.012 \\
& -0.009 \\
& -0.014
\end{aligned}
$$

Diff. W. - O. Rd. künstl. L.

$$
\begin{aligned}
& -0.007 \\
& +0.001 \\
& +0.001 \\
& -0.003
\end{aligned}
$$

Mittel für Venus-Linien $\quad=-0$ R० 7 Mittel für künstliche Linien $=-0.002$ Verschiebung ( $\mathrm{W}-\mathrm{O}$ ) Rand $=-0.015$

$$
2.5 v=-2.7 \mathrm{~km} \quad v=-1.1 \mathrm{~km} \text {. }
$$

April 6. Expositionszeit 60 Minuten.

$\begin{array}{cc}\lambda & \text { Pos. }- \text { Kr. Venus-L. } \\ 424 \mu \mu & \text { I } 5 \% 0 \\ 426 & 14.0 \\ 428 & 15.5 \\ 431.5 & 14.0 \\ H \gamma & 14.5 \\ 435.3 & 14.8 \\ 436.0 & 15.4 \\ 437.1 & 14.8 \\ 439.5 & 14.4 \\ 440.5 & 14.5 \\ 441.5 & 14.4 \\ 442.6 & 14.2 \\ 443.5 & 14.6\end{array}$

Mittel für Venus-Linien $=14 \% 7$

Mittel für künstliche Linien $=14.1$

$$
\varphi-\varphi_{0}=-0.6
$$

Apri] 7. Expositionszeit 60 Minuten.

$\begin{array}{cc}\lambda & \text { Pos. - Kr. Venus-L. } \\ 425.1 \mu \mu & 12.72 \\ 426.1 & 12.62 \\ 427.5 & 12.46 \\ 429.4 & 13.08 \\ 431.5 & 12.88 \\ 435.2 & 12.68 \\ 436.8 & 12.46 \\ 440.5 & 12.68 \\ 446.5 & 12.76 \\ 452 & 12.35 \\ 452.5 & 12.54 \\ \text { Mittel } & \text { I } 2.66 \\ \lambda & \text { Pos. - Kr. kinstl. L. } \\ 427.2 \mu \mu & 12.74 \\ 430.8 & 12.82 \\ 438.4 & 12.75 \\ \text { Mittel } & 12.77 \\ \varphi-\varphi_{0}= & +0.11 \\ +0.6 \mathrm{~km} & v=+0.3 \mathrm{~km} \text { pro Sec. }\end{array}$

April 8. 1. Platte, Expositionszeit 7 Minuten.

$\begin{array}{lc}\quad & \text { Pos. }- \text { Kr. Venus-L. } \\ 425.1 \mu \mu & \text { I } 3.02 \\ 427.5 & \text { I } 3.16 \\ 429 & 12.60 \\ 430.7 & \text { I } 2.72 \\ 431.5 & \text { I } 2.90 \\ 435.2 & \text { I } 2.94 \\ 438.4 & \text { I } 2.82 \\ 440.5 & \text { I } 2.40 \\ 445.9 & \text { I } 2.80 \\ \text { Mittel } & \text { I } 2.82\end{array}$

$\begin{array}{cc}\lambda & \text { Pos. }- \text { Kr. künstl. L. } \\ 427.2 \mu \mu & \text { I } 3.02 \\ 430.8 & \text { I } 3.02 \\ 432.6 & \text { I } 3.02 \\ 438.4 & \text { I } 3.06 \\ 440.5 & \text { I } 3.10 \\ \text { Mittel } & \text { I } 3.04\end{array}$

$\varphi-\varphi_{0}=+0.22$
$2 v=+1.38 \mathrm{~km} v=+0.7 \mathrm{~km}$ pro Sec.

April 8. 2. Platte, zwei Aufnahmen zu 7 Min. Klünstliche $H \gamma$-Linie in der Mitte unterbrochen. I. Aufnahme.

$\begin{array}{cc}\lambda & \text { Pos. - Kr. Venus-L. } \\ 423.6 \mu \mu & 12.06 \\ 431.5 & 12.88 \\ 432.6 & 12.18 \\ 435.2 & 12.56 \\ 436.0 & 12.82\end{array}$




$\begin{array}{lc}\lambda & \text { Pos. - Kr. Venus-L. } \\ 440.5 \mu \mu & 12.82 \\ 441.5 & 12.70 \\ \text { Mittel } & 12.53 \\ \text { Künstl. } H \gamma \cdot \mathrm{L} . & 12.97 \\ \varphi-\varphi_{0}=+0.44 \\ +2.8 \mathrm{~km} v=+1.4 \mathrm{~km} \text { pro Sec. }\end{array}$

April 8. 2. Aufnahme.

\begin{tabular}{|c|c|}
\hline$\lambda$ & Pos. - Kr. Venus-L. \\
\hline $425.1 \mu \mu$ & $11 \% 1$ \\
\hline $427 \cdot 5$ & II. 14 \\
\hline 429.5 & 11.26 \\
\hline 31.5 & 12.30 \\
\hline 32.6 & 12.13 \\
\hline 435.2 & 12.28 \\
\hline 436.0 & 12.06 \\
\hline $441 \cdot 5$ & 11.90 \\
\hline Mittel & I $3.8 \mathrm{I}$ \\
\hline Künstl. $H \gamma \cdot \mathrm{L}$. & 12.24 \\
\hline
\end{tabular}

April Io. I. Platte, Exp.Z. 8 Min., keine gute Platte.

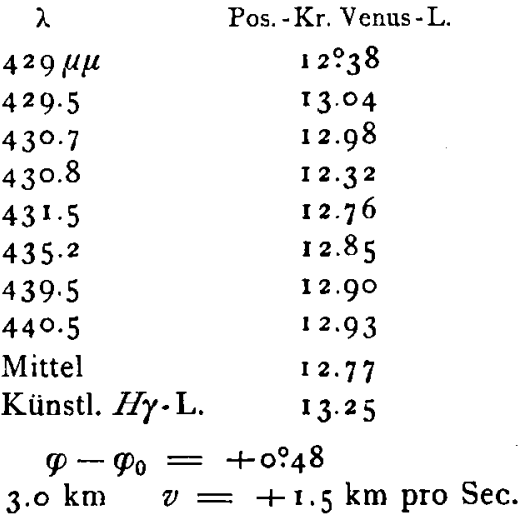

Die zwei anderen Platten eignen sich nicht zu feinen Ausmessungen.

April Ix. 2 Aufnahmen auf einer Platte. Expositionszeit 8 Minuten. r. Aufnahme.

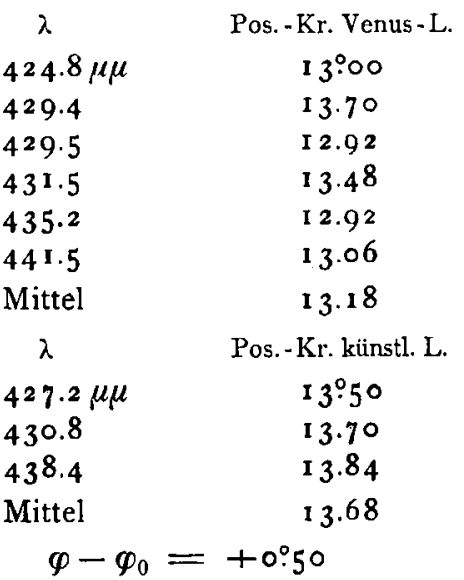

April I I. 2. Aufnahme.

$\begin{array}{lc}\lambda & \text { Pos. - Kr. Venus-L. } \\ 424.8 \mu \mu & 13.08 \\ 428 & \text { I } 2.70 \\ 431.5 & \text { 13.16 } \\ 441.5 & 13.32 \\ \text { Mittel } & \text { 1 } 3.03 \\ \lambda & \text { Pos. - Kr. künsti. L. } \\ 427.2 \mu \mu & \text { I } 2.90 \\ 430.8 & \text { 1 } 2.98 \\ 438.4 & \text { I } 3.14 \\ \text { Mittel } & \text { I } 3.01 \\ \varphi-\varphi_{0}=-0.02\end{array}$

Mittel aus beiden Aufnahmen:

$$
\varphi-\varphi_{0}=+0.24
$$

$2 v=+1.5 \mathrm{~km} v=+0.8 \mathrm{~km}$ pro Sec.

April 20. Expositionszeit 7 Minuten.

$\begin{array}{cc}\lambda & \text { Pos. - Kr. Venus-L. } \\ 425.1 \mu \mu & 14.5 \\ 427.5 & 14.3 \\ 428.8 & 13.1 \\ 429.4 & 13.6 \\ 431.5 & 13.7 \\ \text { Mittel } & 13.76 \\ \lambda & \text { Pos. } \text { Kr. künstl. L. } \\ 427.5 \mu \mu & 13.92 \\ 430.8 & 13.88 \\ 438.4 & 13.82 \\ 440.5 & 13.72 \\ \text { Mittel } & 13.74 \\ \varphi-\varphi_{0} & -0.02\end{array}$

April 20. Der Spalt ist senkrecht zur täglichen Bewegung dicht am Terminator gehalten.

\begin{tabular}{|c|c|}
\hline$\lambda$ & Pos. - Kr. Venus-L. \\
\hline $420.2 \mu \mu$ & $13^{\circ}: 1$ \\
\hline 420.7 & I 2.3 \\
\hline $42 \mathrm{I} .1$ & I 2.6 \\
\hline 42 I. 6 & 13.0 \\
\hline 421.9 & I 3.5 \\
\hline 423.0 & I $3 \cdot 5$ \\
\hline 423.7 & 12.9 \\
\hline 424.0 & 12.7 \\
\hline 425.1 & I 3.0 \\
\hline $425 \cdot 5$ & 13.1 \\
\hline 426.1 & 13.3 \\
\hline $427 \cdot 5$ & 12.4 \\
\hline 429.2 & 13.5 \\
\hline $4^{29} \cdot 4$ & I 3.2 \\
\hline 430.7 & $13 \cdot 3$ \\
\hline 430.8 & 13.1 \\
\hline Mittel & I 3.03 \\
\hline$\lambda$ & Pos. -Kr. künstl. L \\
\hline $427.2 \mu \mu$ & $12: 98$ \\
\hline 430.8 & 13.01 \\
\hline $45^{2 \cdot 5}$ & 12.92 \\
\hline Mittel & 12.97 \\
\hline$\varphi-$ & $-0: 06$ \\
\hline
\end{tabular}


Dieselbe Platte. Zweite Messung.

$\begin{array}{cc}\lambda & \text { Pos. - Kr. Venus-L. } \\ 418.8 \mu \mu & 13.65 \\ 420.7 & 13.26 \\ 421.0 & 13.66 \\ 421.6 & 13.50 \\ 421.9 & 14.00 \\ 423.6 & 13.66 \\ 425.1 & 13.38 \\ 425.5 & 13.68 \\ 427.5 & 13.86 \\ 429.4 & \text { I } 3.96 \\ \text { Mittel } & \text { I } 3.66 \\ \lambda & \text { Pos. - Kr. künstl. L. } \\ 427.2 \mu \mu & 13.63 \\ 430.8 & \text { I } 3.56 \\ \text { Mittel } & \text { I } 3.60 \\ \varphi-\varphi_{0}= & -0.06\end{array}$

April 20. 2. Platte, Spalt parallel dem Terminator.

\begin{tabular}{|c|c|}
\hline${ }_{4 \circ}^{\lambda} 8^{2} . \mu \mu$ & $\begin{array}{c}\text { Pos. - Kr. Venus-L. } \\
\text { r } 3: 8\end{array}$ \\
\hline $4 \times 3.2$ & I 2.8 \\
\hline 414.4 & I $3 \cdot 3$ \\
\hline $4 \times 4.9$ & I 3.9 \\
\hline 417.1 & I $3 \cdot 7$ \\
\hline 417.4 & 14.0 \\
\hline 417.6 & I 3.1 \\
\hline 418.7 & 13.1 \\
\hline 418.8 & 13.5 \\
\hline 421.1 & 13.2 \\
\hline 421.6 & 13.8 \\
\hline $424 \cdot 3$ & 13.6 \\
\hline 424.5 & 13.7 \\
\hline 425.1 & 13.7 \\
\hline $\begin{array}{l}427 \cdot 5 \\
\text { Mittel }\end{array}$ & $\begin{array}{l}13.4 \\
13.48\end{array}$ \\
\hline$\lambda$ & Pos. -Kr. kiünstl. L. \\
\hline $427.2 \mu \mu$ & $13{ }^{\circ} 49$ \\
\hline 430.8 & 13.62 \\
\hline Mittel & 1 $3.5^{6}$ \\
\hline
\end{tabular}

April 2o. 3. Platte, Spalt parallel dem Terminator, violettes Ende schwach.

$\begin{array}{cc}\lambda & \text { Pos. }- \text { Kr. Venus-L. } \\ 424.0 \mu \mu & 13.7 \\ 425.1 & \text { I } 3.9 \\ 427.5 & \text { I } 3.4 \\ 429.4 & 13.7 \\ 430.7 & 13.6 \\ 435.2 & \text { I } 3.7 \\ 435.3 & \text { I } 4.1 \\ 436.0 & \text { I } 4.2 \\ 437.1 & 13.6 \\ 439.5 & \text { I } 4.1 \\ 441.5 & 14.0 \\ 443.5 & 14.1 \\ \text { Mittel } & \text { I } 3.84\end{array}$

$\begin{array}{lc}\lambda & \text { Pos. - Kr. künstl. L. } \\ 427.2 \mu \mu & \text { I } 3.90 \\ 430.8 & \text { I } 3.87 \\ 432.6 & 14.03 \\ 438.4 & 13.97 \\ 440.5 & \text { I } 3.96 \\ \text { Mittel } & \text { I } 3.95 \\ \varphi-\varphi_{0}=+0.11\end{array}$

April 28. Spalt parallel dem Terminator.

$\begin{array}{cc}\lambda & \text { Pos. - Kr. Venus - L. } \\ 425.1 \mu \mu & 13^{\circ} .44 \\ 425.5 & 13.06 \\ 427.5 & 13.63 \\ 429.4 & \text { I } 3.48 \\ 430 & 13.18 \\ 430.8 & 13.32 \\ 431.5 & 13.43 \\ 435.2 & 13.48 \\ \text { Mittel } & 13.3^{8} \\ \lambda & \text { Pos. - Kr. künstl. L. } \\ 427.2 \mu \mu & \text { I } 3^{\circ} .33 \\ 430.8 & 13.47 \\ 432.6 & 13.43 \\ 43^{8} .4 & 13.42 \\ \text { Mittel } & 13.4 \text { I } \\ \varphi-\varphi_{0}= & +0.03\end{array}$

Stellen wir alle $\varphi-\varphi_{0}$ zusammen, so erhalten wir für den Fall, wenn der Spalt parallel zur täglichen Bewegung gestellt war:

$$
\begin{aligned}
& \text { März } 30+0.05 \text { April } 8+0.43 \\
& 230+0.50 \text { \$ } 10+0.48 \\
& \text { April } 6-0.6 \text { I I }+0.50 \\
& \text { } 7+0.11 \text { i I }-0.02
\end{aligned}
$$

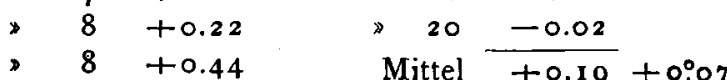

Die Werthe von $\varphi-\varphi_{0}$ in dem Falle, wenn der Spalt parallel zum Terminator gestellt war, sind folgende:

$$
\begin{aligned}
& \text { April } 20-0.06 \text { April } 20 \quad+0.111 \\
& \text { } 20-0.06 \\
& \text { - } 20+0.08 \quad \text { Mittel } \frac{0.00}{0.03}
\end{aligned}
$$

Wenn man den Werth $\varphi-\varphi_{0}=+0.19$ als reell annehmen will, so kann man den Werth der Geschwindigkeit des Venusaequators berechnen. Der lineare Halbmesser der Venus war in der Focalebene des $3 \circ$ Zöllers $=0.6 \mathrm{~mm}$ $=9: 3$. Da aber die Focallänge des Camera-Objectivs sich zu der Focallänge des Collimator-Objectivs wie 2 zu 3 verhält, so ist der Halbmesser in der Ebene der Platte gleich $0.4 \mathrm{~mm}$ gleich 1.60 Revolutionen der Schraube.

Auf diese Weise erhalten wir

$$
\begin{gathered}
2 v=K R \sin \left(\varphi_{0}-\varphi\right)=1.2 \mathrm{~km} \text { pro Sec. } \\
v=+0.6 \mathrm{~km} \text { pro Sec. } \pm 0.3 \mathrm{~km} .
\end{gathered}
$$

Es bleiben dann noch die unmittelbar erhaltenen Geschwindigkeiten : 


$\begin{array}{ccc}\text { März } 25 & +1.6 \mathrm{~km} \\ \gg & 25 & +1.3 \\ \text { April }^{2} & 30 & +0.4 \\ & 4 & -1.1\end{array}$

Mittel $=+0.55 \mathrm{~km} \pm 0.41 \mathrm{~km}$ pro Sec.

Weiter folgen Messungen der Platten, welche mittelst des Dreiprismen-Spectrographen erhalten worden sind.

Mai 4. I. Aufnahme, Expositionszeit 30 Minuten.

$\begin{array}{cc}\lambda & \text { Pos. }- \text { Kr. Venus-L. } \\ 435.2 \mu \mu & 12.78 \\ 436.0 & 12.70 \\ 436.5 & 12.60 \\ 436.7 & 12.92 \\ 436.8 & 12.80 \\ 437.1 & 12.36 \\ 439.5 & 12.10 \\ 441.0 & 12.49 \\ 441.5 & 12.48 \\ 442.7 & 12.76 \\ 445.5 & 12.74 \\ 445.9 & 12.80 \\ \text { Mittel } & 12.63 \\ \lambda & \text { Pos. }- \text { Kr. künstl. L. } \\ 438.4 \mu \mu & 12.96 \\ 440.5 & 12.96 \\ 452.5 & 13.02 \\ \text { Mittel } & 12.98 \\ \varphi-\varphi_{0} & +0.35\end{array}$

Mai 4. Dieselbe Platte. Zweite Messung.

\begin{tabular}{|c|c|}
\hline$\lambda$ & Pos.-Kr. Venus-L. \\
\hline $435.2 \mu \mu$ & $12 \% 20$ \\
\hline 436.0 & 12.66 \\
\hline $43^{6.8}$ & I 1.77 \\
\hline 437.0 & 11.40 \\
\hline $439 \cdot 3$ & I 1.00 \\
\hline 439.5 & 10.72 \\
\hline 441.0 & 11.74 \\
\hline $441 \cdot 5$ & 11.76 \\
\hline 442.7 & I I. 54 \\
\hline $445 \cdot 5$ & II.50 \\
\hline 445.9 & I I $\cdot 39$ \\
\hline 446 & 11.82 \\
\hline 452.7 & 1 1.96 \\
\hline 453 & I I. 54 \\
\hline Mittel & I 1.64 \\
\hline$\lambda$ & Pos. - Kr. künstl. L. \\
\hline $43^{8.4 \mu \mu}$ & I $1: 78$ \\
\hline 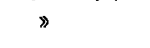 & I 1.88 \\
\hline 440.5 & I 1.48 \\
\hline 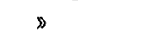 & 11.92 \\
\hline $45^{2.5}$ & 12.05 \\
\hline 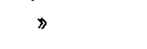 & I I.98 \\
\hline Mittel & 11.85 \\
\hline$\varphi-\varphi$ & +0.21 \\
\hline
\end{tabular}

Mai 4. 2. Aufnahme, Expositionszeit 38 Minuten.

\begin{tabular}{|c|c|}
\hline$\lambda$ & Pos. -Kr. Venus-L \\
\hline $437.0 \mu \mu$ & $10: 94$ \\
\hline 437.1 & 10.80 \\
\hline 439.5 & 10.80 \\
\hline 440.5 & I I. I 8 \\
\hline 441.0 & I I .04 \\
\hline $44 I .5$ & 11.22 \\
\hline 442.7 & 11.55 \\
\hline $444 \cdot 3$ & 10.90 \\
\hline $445 \cdot 5$ & I I $44^{\circ}$ \\
\hline 445.9 & I I .37 \\
\hline 452.9 & I 123 \\
\hline 455.0 & 11.43 \\
\hline Mittel & I I I I 6 \\
\hline$\lambda$ & Pos. - Kr. künstl. L. \\
\hline $43^{8.4 \mu \mu}$ & $11: 52$ \\
\hline$\nexists$ & 11.90 \\
\hline 452.5 & 11.76 \\
\hline Mittel & I I.73 \\
\hline$\varphi-$ & +0.57 \\
\hline
\end{tabular}

Dieselbe Platte. Zweite Messung.

\begin{tabular}{|c|c|}
\hline$\lambda$ & Pos. - Kr. Venus-L. \\
\hline $435.2 \mu \mu$ & 12928 \\
\hline $43^{6.0}$ & 19.82 \\
\hline 436.8 & 11.76 \\
\hline 437.1 & $11.5^{8}$ \\
\hline 439.5 & 11.12 \\
\hline 441.0 & I 1.94 \\
\hline 441.5 & I 2.14 \\
\hline 442.7 & 12.16 \\
\hline 444 & I 1.96 \\
\hline $445 \cdot 5$ & 11.72 \\
\hline $445 \cdot 9$ & 11.98 \\
\hline Mittel & 11.85 \\
\hline$\lambda$ & Pos.-Kr. kiinstl. L. \\
\hline $43^{8.4} \mu \mu$ & $12 \% 10$ \\
\hline » & I 2.14 \\
\hline$\triangleright$ & 12.23 \\
\hline 452.5 & I 2.40 \\
\hline Mittel & I 2.2 I \\
\hline
\end{tabular}

Mai 4. 3. Aufnahme, Expositionszeit 35 Minuten.

$\begin{array}{cc}\lambda & \text { Pos. }- \text { Kr. Venus - L. } \\ 441.5 \mu \mu & 10.18 \\ 442.7 & 10.14 \\ 444.3 & 10.52 \\ 446.5 & 10.58 \\ 447.6 & 11.33 \\ 45^{2.3} & 11.44 \\ \text { Mittel } & 10.70 \\ \lambda & \text { Pos. }- \text { Kr. künstl. L. } \\ 43^{8.4} \mu \mu & 10.98 \\ \Rightarrow & 11.05 \\ 440.5 & 11.02\end{array}$




$\begin{array}{lc}\lambda & \text { Pos. }- \text { Kr. kiunstl. L. } \\ 440.5 \mu \mu & 11.006 \\ 45^{2.5} & 11.04 \\ \text { Mittel } & 11.03 \\ \varphi-\varphi_{0}=+0.33\end{array}$

Mai 5. Expositionszeit 5o Min., überexponirte Platte, Linien unscharf.

$\begin{array}{cc}\lambda & \text { Pos. - Kr. Venus-L } \\ 434.1 \mu \mu & 11.29 \\ 435.2 & 11.27 \\ 436.0 & 11.27 \\ 436.8 & 11.21 \\ 437.1 & 11.37 \\ 439.5 & 11.26 \\ 442.7 & 11.24 \\ 443.5 & 12.14 \\ 445.5 & 11.50 \\ \text { Mittel } & 11.45 \\ \lambda & \text { Pos. - Kr. kïnstl. L. } \\ 438.4 \mu \mu & 12.04 \\ \nu & 12.04 \\ 440.5 & 12.02 \\ \nu & 11.97 \\ \text { Mittel } & 12.02 \\ \varphi-\varphi_{0} & +0.57\end{array}$

Mai 13. Wegen rascher Aenderung der Temperatur verwaschen.

\begin{tabular}{|c|c|}
\hline$\lambda$ & Pos.-Kr. Venus-L. \\
\hline$\$ 35.2 \mu \mu$ & 11970 \\
\hline 436.0 & 11.60 \\
\hline $43^{6.8}$ & II.74 \\
\hline 37.0 & 11.98 \\
\hline $437 \cdot 1$ & 12.70 \\
\hline+39.5 & 13.70 \\
\hline 440.0 & 12.82 \\
\hline 442.7 & 11.94 \\
\hline Mittel & I 2.27 \\
\hline$\stackrel{\lambda}{\lambda}{ }_{3.4 \mu \mu}$ & $\begin{array}{l}\text { Pos. - Kr. künstl. L. } \\
\text { I } 2: 47\end{array}$ \\
\hline 440.5 & 12.60 \\
\hline ittel & I 2.54 \\
\hline$\varphi-\varphi$ & +0.27 \\
\hline
\end{tabular}

Pulkowo, Mai I 900.
Die Zusammenstellung der $\varphi-\varphi_{0}$ giebt:

$$
\begin{array}{ccc}
\text { Mai } & 4 & +0.35 \\
\Rightarrow & 4 & +0.21 \\
\Rightarrow & 4 & +0.57 \\
\Rightarrow & 4 & +0.36 \\
\Rightarrow & 4 & +0.33 \\
\Rightarrow & 5 & +0.57 \\
\Rightarrow & 13 & +0.27 \\
\cline { 2 - 2 } & \text { Mittel } & +0.38 \pm 0.04
\end{array}
$$

Der Venushalbmesser war zu dieser Zeit im Mittel gleich I I."o, was in der Ebene der Platte $0.5 \mathrm{~mm}=\mathbf{2} . \mathbf{R}_{0}$ entspricht. Die resultirende Geschwindigkeit ist also

$2 v=1.8 \mathrm{~km} \cdot v=+0.9 \mathrm{~km}$ pro Sec. $\pm 0.1 \mathrm{~km}$.

Diese mittelst wenig zum Zweck geeigneter Instrumente erhaltenen Resultate sollen nur als Versuch die Rotationsgeschwindigkeit der Venus zu bestimmen, angesehen werden.

Die modernen mächtigen Instrumente von Potsdam, der Lick- und Yerkes-Sternwarte etc. können leicht meine Resultate bestätigen oder widerlegen, jedenfalls die Frage lösen.

Aus den obigen Untersuchungen könnte man schliessen, dass Venus in einer Zeit von etwa einem Tag rotire.

Um die Rotationszeit auf eine Stunde genau zu ermitteln, mitssen Spectrographen angewandt werden, welche die Geschwindigkeit im Visionsradius aus einer Platte mit einem w. F. von $\pm 0.1 \mathrm{~km}$ pro Sec. bestimmen können.

Wenn wir den Venusdurchmesser $z u=12700 \mathrm{~km}$ annehmen, so entspricht einer Aequatorgeschwindigkeit von $v$ eine Rotationszeit $T$ :

$$
\begin{array}{lllll}
v=0.7 \mathrm{~km} & 0.5 \mathrm{~km} & 0.462 \mathrm{~km} & 0.45 \mathrm{~km} & 0.3 \mathrm{~km} \\
T=15^{\mathrm{h}} .9 & 22^{\mathrm{h}} \mathrm{I} & 24^{\mathrm{h}} .0 & 24^{\mathrm{b}} .6 & 37^{\mathrm{h}} .0
\end{array}
$$

Ich muss gestehen, dass ich bei den Messungen unwillkürlich diejenigen Spectrallinien wählte, welche eine Verschiebung im erwünschten Sinne unzweifelhaft zeigten. Von diesem Fehler konnte ich mich kaum frei machen. Vielleicht ist dadurch der immer zu grosse Werth der Aequatorgeschwindigkeit, den ich an beiden Instrumenten gefunden habe, zu erklären.

Da das schlechte Wetter und die schmale Sichel der Venus uns nicht erlauben, die Untersuchungen in diesem Jahre fortzuführen, so sind die Beobachtungen mit dem 13. Mai geschlossen.

\section{A. Belopolsky.}

Neuer Planet 1900 FG.

Planet Wolf-Schwassmann 22. Mai $\quad 2^{\mathrm{h}} 9^{\mathrm{m}} \cdot 7$ M. Z. Königstuhl RA. $=23^{\circ} 6^{\circ}$ PD. $=1 \circ 3^{\circ} 25^{\prime}$ tägl. Bew. - I $3^{\prime} \circ^{\prime}$ Gr. r r.9. Wolf.

Der Planet ist auf der Sternwarte des Collegio Romano wie folgt beobachtet: 24. Mai I I ${ }^{\mathrm{h}} 34^{\mathrm{m}_{7}}$ M. Z. Rom RA. app. $=235^{\circ} 44^{\prime} 39^{\prime \prime}$ PD. app. $=103^{\circ} 26^{\prime} 9^{\prime \prime}$ Gr. 1 1.o. Millosevich.

Berichtigung zu Nr. 3636 Bd. 152 p. 192 Z. 31 v. o. statt: where he ... buried lies: he as well as Mrs. Smyth are buried in the churchyard of Sharow, in which parish Clova is situated.

Inhalt zu Nr. 3640-4I. A. Abetti. Asteroidi osservati ad Arcetri nel 1899. 245. - M. W. Whitney. Occultations observed during the Lunar eclipse of Dec. 16, 1899. 26I. - A. Belopolsky. Ein Versuch die Rotationsgeschwindigkeit des Venusaequators auf spectrographischem Wege za bestimmen. 263. - Neuer Planet I900 FG. 275. - Berichtigung. 275. 